inactivation? It is tempting to suggest $\mathrm{PKC} \zeta$, but there is currently no direct biochemical evidence for this. Which other Cdc42 targets could be involved in membrane protrusion initiation? Cdc42 is necessary to form protrusions, whereas the other components of the pathway are not (Fig. 1). Instead, PKCל and GSK-3 are required to regulate the orientation of protrusions towards the wound (Fig. 1). So, which other additional signalling pathways control the positioning of protrusions? Is APC also involved?

At a more general level, $\mathrm{Cdc} 42$ is now a hot candidate for regulating microtubule dynamics $^{1,12}$. Thus, Cdc42 and the other family members Rho and Rac can regulate the dynamics of both microtubule and intermediate filaments ${ }^{13,14}$, functions beyond their established role in actin cytoskeleton.

To reorganize the MTOC in migrating astrocytes, polarization signals initiated by Cdc42-Par6-PKCל use GSK-3 and APC, two components of the Wnt pathway. Clearly, astrocyte migration need not require a Wnt signal to generate the polarizing signal. Could two independent pathways that regulate polarization use the same proteins to modulate microtubules (Figs 1, 2)? Or could it be that Cdc42 and Wnt pathways interact with each other? Interestingly, a similarity between Cdc42-Par6-PKC $\zeta$ and unconventional Wnt signal pathways is that $\beta$-catenin is not required for pathways that result in reorganization of microtubules. Could the formation of a complex between Par6-PKC $\zeta$ and GSK-3 represent an alternative mode of $\beta$-catenin and APC regulation? Obviously, it will be interesting to re-examine Wnt signalling for an interaction with $\mathrm{Cdc} 42$ and PKCל (and vice versa). Whatever the answers to these questions, the new results derived from astrocyte migration certainly point us in new directions for unravelling the regulation of cell polarity by Cdc42 and Wnt.

Adrain Harwood is in the MRC Laboratory of Molecular Cell Biology

\& Department of Biology, University College

London, Gower Street, London, WC1E 6BT.
Vania M. M. Braga is in the Cell and Molecular Biology Section, Division of Biomedical Sciences, Faculty of Medicine, Imperial College London, Sir Alexander Fleming Building, London

e-mail:v.braga@imperial.ac.uk

1. Etienne-Manneville, S. \& Hall, A. Nature 421, 753-756 (2003)

2. Ahringer, J. Curr. Opin. Cell Biol. 15, 73-81 (2003).

3. Etienne-Manneville, S. \& Hall, A. Nature 420, 629-635 (2002).

4. Shi, S. H., Jan, L. Y. \& Jan, Y. N. Cell 112, 63-75 2002

5. Etienne-Manneville, S. \& Hall, A. Cell 106, 489-496 (2001).

6. Magdalena, J., Millard, T. H. \& Machesky, L. M. J. Cell Sci. 116, 743-756 (2003).

Lucas, F., Goold, R., Gordon-Weeks, P. \& Salinas, P. J. Cell Sci. 111, 1351-1361 (1998).

8. Zumbrunn, J., Kinoshita, K., Hyman, A. A. \& Nathke, I. S. Curr. Biol. 11, 44-49 (2001).

9. Schlesinger, A. et al. Genes Dev. 13, 2028-2038 (1999).

10. Wakefield, J. G., Stephens, D. J. \& Tavare, J. M. J. Cell Sci. 116 , 637-646 (2003).

11. Fodde, R. et al. Nature Cell Biol. 2, 433-438 (2001).

12. Palazzo, A. F. et al. Curr. Biol. 11, 1536-1541 (2001).

13. Gundersen, G. G. \& Cook, T. A. Cur. Opin. Cell Biol. 11, 81-94 (1999)

14. Braga, V. M. M. Curr. Opin. Cell Biol. 14, 546-556 (2002).

\section{Leading the pack}

The ability of cells to polarize and migrate up a chemical gradient is key to a variety of processes, from axon guidance to leukocyte homing. This ability to chemotax is also key to the survival of the social amoebae Dictyostelium discoideum during times of starvation, and studies in this system have provided important insights into the molecular mechanisms directing chemotaxis. One key question in the field is how migrating cells amplify chemo-attractant gradients, and new work published by Kriebel et al. (Cell 112, 549-560 (2003)) now provides a model for this. They show that $D$. discoideum cells amplify the gradient of the chemo-attractant cyclic AMP by asymmetrically localizing the enzyme responsible for its generation.

On exposure to a cAMP chemo-attractant gradient, D. discoideum cells polarize, begin to migrate towards the gradient source, and then line up head-to-tail in streams to form aggregates that are key to their survival. Now, Kriebel et al. propose that by localizing adenylyl cyclase (ACA) - the enzyme responsible for generating cAMP - to the uropod during polarization, migrating cells can then themselves produce cAMP from their tail. In this way, they provide an amplified cAMP signal for the cells behind to follow.

Cells lacking ACA can polarize when exposed to a chemoattractant gradient, but cannot then line up to form streams. To see where ACA localizes in the cell, the authors fused ACA to yellow fluorescent protein (YFP) and found that after polarization, ACA is highly enriched at the uropod. Intriguingly, ACA is also present in intracellular vesicles, suggesting that vesicular transport may be involved in its localization or in the signal transduction pathways that result in its activation.

So what is the pathway that mediates ACA localization? Neither CRAC (cytosolic regulator of adenylyl cyclase), which activates ACA, nor PKA (protein kinase A), a target of ACA, seem to be involved. By perturbing the actin cytoskeleton with drug treatments, the authors find that the actin cytoskeleton is important for ACA localization, but the exact mechanism by which this takes place remains to be established. The enzymatic activity of ACA also affects its localization, as cells expressing a

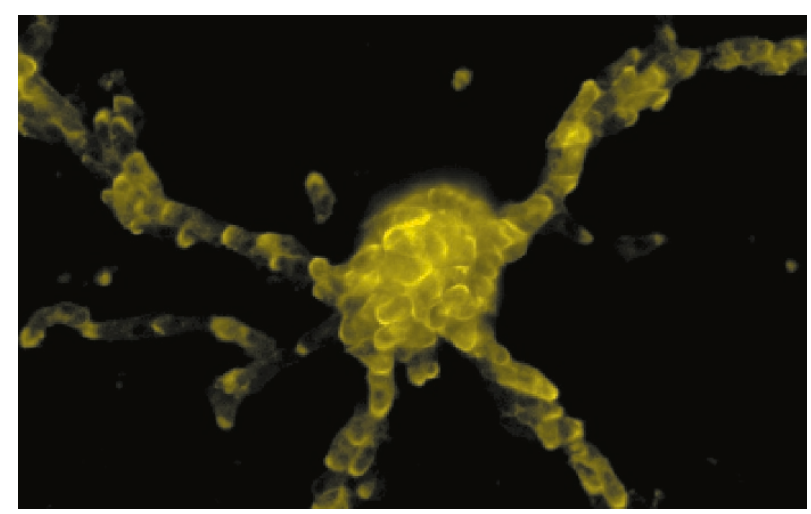

D. discoideum amobae chemotax towards a gradient source. As they migrate, $D$. discoideum line up in a head-to-tail fashion to form aggregates. Imaging ACA-YFP reveals plasma membrane labelling that is highly enriched at the uropod of polarized cells. Image provided by C. Parent.

constitutively active mutant of ACA are also unable to stream.

The importance of this work is that it suggests an amplifying mechanism by which migrating cells responding to a chemoattractant can then themselves become a source of chemoattractant for other cells to follow. This signal relay may also be common to other mammalian cell types. In leukocytes, for example, it is known that migrating cells secrete chemo-attractant in response to chemo-attractant exposure. This work, therefore, may provide a basis for future studies into the relay mechanisms used by diverse chemotactic cell types.

ALISON SCHULDT 